\title{
Tailoring Surgical Therapy for Extremity Soft Tissue Sarcoma
}

\author{
Steven C. Katz, MD, FACS ${ }^{1,2}$ \\ ${ }^{1}$ Division of Surgical Oncology, Department of Surgery, Roger Williams Medical Center, Providence, RI; ${ }^{2}$ Department of \\ Surgery, Boston University School of Medicine, Boston, MA
}

Limb-sparing multimodality therapy for extremity soft tissue sarcoma (ESTS) affords excellent disease control and functional outcomes in the majority of patients. ${ }^{1}$ In a study of 4977 extremity and superficial trunk STS, 20$25 \%$ experienced recurrence, with some recurrent events occurring more than two decades after initial treatment. ${ }^{2}$ Determining the optimal surgical approach to minimize the risk of ESTS recurrence is a complex undertaking due to extreme biologic heterogeneity, with more than 100 histologic subtypes. ${ }^{3}$ The consideration of ESTS as a single entity is a matter of necessity rather than reason. The wide range of ESTS biologic behavior makes it exceedingly difficult to define broad policies or recommendations for most management dilemmas. ${ }^{4}$

Bonvalot et al. conducted a retrospective study of 541 ESTS patients to examine the minimum necessary margin and the issue of mandatory re-excision followed unplanned resections. ${ }^{5}$ As stated in the title, the authors also set out to examine the relationship between local control of ESTS and survival. The lack of a causal link between ESTS local control and survival has been thoroughly verified previously and, as such, will not be the focus of this editorial. ${ }^{6}$ Among all patients, $82 \%$ underwent R0 resection and the median margin was $2 \mathrm{~mm}$. The median follow-up of 7 years allows for reasonable survival and recurrence estimates. Local recurrences (LR) were experienced by $8 \%$ and $20 \%$ of patients died from all causes. Predictors of worse OS were grade, leiomyosarcoma subtype, and presence of residual disease upon re-excision. Importantly, $47 \%$ of patients were treated following unplanned excision at other hospitals; $58 \%$ of those patients had residual

(C) Society of Surgical Oncology 2016

First Received: 8 July 2016;

Published Online: 1 August 2016

S. C. Katz, MD, FACS

e-mail: skatz@chartercare.org disease upon re-excision. LR was predicted by specific subtypes, such as myxofibrosarcoma, and a margin less than $1 \mathrm{~mm}$.

The impact of residual disease, likelihood of LR occurring before death due to distant metastases, and hence importance of re-excision after unplanned ESTS resection will vary by histologic subtype. In the present study, $22 \%$ of patients had leiomyosarcoma, a disease in which distant recurrence predominates, with only $11 \%$ experiencing isolated LR. ${ }^{7}$ Fewer than $10 \%$ of patients had subtypes with highly aggressive LR patterns, including myxofibrosarcoma and dermatofibrosarcoma protuberans. Whereas deferring re-excision following an unplanned lower-extremity leiomyosarcoma resection may be appropriate in selected cases given that the outcome is driven by distant recurrence, omission in a myxofibrosarcoma patient would generally be inadvisable. As acknowledged by the authors, consideration of subtype biologic behavior is a critical factor when deciding if re-excision following unplanned ESTS surgery is warranted.

The goal of reoperation following unplanned ESTS resection is to eradicate potential residual disease, prevent LR, and improve the likelihood of limb salvage. The majority of unplanned ESTS resections in the present study were indeed inadequate, because $58 \%$ of patient who underwent re-excision were found to harbor residual sarcoma. The rate of residual disease following unplanned ESTS resection is consistent with a range of $45-53 \%$ from prior reports. ${ }^{8-10}$ Despite the present study not demonstrating a significant association between residual tumor and LR, other groups have reported that initial unplanned ESTS resection and residual disease predicted $\mathrm{LR}^{8,9}$ In a study of 142 unplanned ESTS resections from Charoenlap, the amputation rate was significantly higher in patients with residual disease (18.5 vs. $1.8 \%, p=0.003$ ). Of note, only $10 \%$ of patients in the Charoenlap study had leiomyosarcoma compared with $22 \%$ in the report from 
Bonvalot. Inclusion of a lower proportion of patients with tumors prone to isolated LR may obfuscate the relationship between residual disease, local control, and limb salvage. Furthermore, confirming that LR has no impact on survival does not lessen the importance of local control, as LR in and of itself is a morbid and potentially limb-threatening event. $^{11}$

Patients with positive microscopic margins following resection are at significantly elevated risk of $\mathrm{LR},{ }^{12}$ as confirmed in the present report. It is unclear why, in the present study, positive margins correlated with LR whereas residual disease did not. Although a median minimum margin of $2 \mathrm{~mm}$ resulted in a very favorable limb salvage rate, it is unclear how this translates into clinical practice. What are the appropriate clinical margins one should take to ensure at least a 2-mm final pathologic margin? Does a minimum margin of $2 \mathrm{~mm}$ along one aspect of the tumor adjacent to the sciatic nerve justify less extensive resection along the remainder of the tumor? Do we avoid re-excision in a myxofibrosarcoma patient with 2-mm margins following unplanned resection, where skip lesions and infiltrative disease are major concerns? Wide margins are desirable where they can be achieved without causing significant functional loss. Narrow, 2-mm margins are acceptable on aspects of the tumor abutting critical neurovascular structures in most cases. Intraoperative decisions about margin aggressiveness and selection of patients for re-excision should be made on the basis of histologic subtype and functional considerations. Without rigorous prospective comparisons between different surgical strategies and margin lengths, it is unclear if we should accept 2-mm margins in all ESTS cases.

A further confounding factor in the present study is that $78 \%$ of patients received XRT. The authors have not determined if neoadjuvant or adjuvant XRT contributed to favorable outcomes among patients who have residual disease following unplanned ESTS resection. Given the known associated between adjuvant XRT and reduced risk of LR, ${ }^{13}$ stratification of the analysis by XRT treatment would be required to define the true impact of re-excision following unplanned surgery, and whether XRT is required to mitigate potential excess LR risk. While the positive impact of XRT on ESTS LR is well documented, so are the long-term issues of edema and functional limb deficits. ${ }^{13}$

The relationship between residual disease and LR following unplanned ESTS resection is unclear when considering all ESTS subtypes in aggregate. Residual disease will most likely impact LR and limb salvage in subtypes not overly prone to distant recurrence. Bonvalot and colleagues are to be commended for emphasizing the importance of ESTS heterogeneity when tailoring management following unplanned excisions. Re-excision following inadequate ESTS surgery should be performed for histologic subtypes at high risk for isolated LR. Acceptance of narrow margins should be based on tumor infiltration and recurrence patterns. Decisions regarding optimal ESTS management are most appropriately driven by assessments of tumor biologic behavior and natural history, keeping in mind the critical importance of minimizing the risks of LR and limb loss.

CONFLICT OF INTEREST The author has declared that no conflict of interest exists.

\section{REFERENCES}

1. Stojadinovic A, Jaques DP, Leung DH, Healey JH, Brennan MF. Amputation for recurrent soft tissue sarcoma of the extremity: indications and outcome. Ann Surg Oncol. 2001;8:509-18.

2. Brennan MF, Antonescu CR, Moraco N, Singer S. Lessons learned from the study of 10,000 patients with soft tissue sarcoma. Ann Surg. 2014;260:416-21. discussion 21-2.

3. Crago AM, Brennan MF. Principles in management of soft tissue sarcoma. Adv Surg. 2015;49:107-22.

4. Feig B, Benjamin R. Guidelines for the treatment of recurrent retroperitoneal sarcoma: are we trying to fit a square peg into a round hole? Ann Surg Oncol. 2016. doi:10.1245/s10434-0165344-7.

5. Bonvalot S, Levy A, Terrier P, Tzanis D, Bellefqih S, La Cesne A, et al. Primary extremity soft tissue sarcomas: does local control impact survival? Ann Surg Oncol. 2016.

6. Brennan MF. Local recurrence in soft tissue sarcoma: more about the tumor, less about the surgeon. Ann Surg Oncol. 2007;14:1528-9.

7. Gladdy RA, Qin LX, Moraco N, Agaram NP, Brennan MF, Singer S. Predictors of survival and recurrence in primary leiomyosarcoma. Ann Surg Oncol. 2013;20:1851-7.

8. Charoenlap C, Imanishi J, Tanaka T, Slavin J, Ngan SY, Chander $\mathrm{S}$, et al. Outcomes of unplanned sarcoma excision: impact of residual disease. Cancer Med. 2016;5:980-8.

9. Qureshi YA, Huddy JR, Miller JD, Strauss DC, Thomas JM, Hayes AJ. Unplanned excision of soft tissue sarcoma results in increased rates of local recurrence despite full further oncological treatment. Ann Surg Oncol. 2012;19:871-7.

10. Zornig C, Peiper M, Schroder S. Re-excision of soft tissue sarcoma after inadequate initial operation. Br J Surg. 1995;82:2789.

11. Ferrone ML, Raut CP. Modern surgical therapy: limb salvage and the role of amputation for extremity soft-tissue sarcomas. Surg Oncol Clin N Am. 2012;21:201-13.

12. Pisters PW, Leung DH, Woodruff J, Shi W, Brennan MF. Analysis of prognostic factors in 1,041 patients with localized soft tissue sarcomas of the extremities. $J$ Clin Oncol. 1996;14:1679-89.

13. Beane JD, Yang JC, White D, Steinberg SM, Rosenberg SA, Rudloff U. Efficacy of adjuvant radiation therapy in the treatment of soft tissue sarcoma of the extremity: 20-year follow-up of a randomized prospective trial. Ann Surg Oncol. 2014;21:2484-9. 